\title{
Cultivo de jundiás alimentados com dietas com casca de soja ou de algodão
}

\author{
Fabio de Araújo Pedron ${ }^{(1)}$, João Radünz Neto(1), Tatiana Emanuelli(2), Leila Picolli da Silva(1), Rafael Lazzari(1), \\ Viviani Corrêia ${ }^{(1)}$, Giovani Taffarel Bergamin ${ }^{(1)}$ e Cátia Aline Veiverberg ${ }^{(1)}$
}

\begin{abstract}
(1)Universidade Federal de Santa Maria (UFSM), Departamento de Zootecnia, Laboratório de Piscicultura, Avenida Roraima, № 1.000, CEP 97105-900 Santa Maria, RS. E-mail: pedron.ufsm@hotmail.com, jradunzneto@smail.ufsm.br, leilasliva@yahoo.com.br, rafaellazzari@yahoo.com.br, vivianicorreia@hotmail.com, giovanitb@gmail.com, catiaveiver@yahoo.com.br (2)UFSM, Departamento de Tecnologia e Ciência dos alimentos. E-mail: etaty@terra.com.br
\end{abstract}

\begin{abstract}
Resumo - O objetivo deste trabalho foi avaliar o efeito da fibra bruta da dieta, no crescimento, parâmetros digestivos, rendimento e qualidade de carcaça de juvenis de jundiá (Rhamdia quelen) em tanques-rede. $\mathrm{O}$ delineamento experimental foi inteiramente casualizado, em arranjo fatorial $2 \times 3$ (duas fontes - cascas de soja $\mathrm{e}$ de algodão - e três níveis de fibra bruta: 4,7 e 10\%), com três repetições. Foram distribuídos 450 peixes $(129,11 \pm 7,8 \mathrm{~g})$ ao acaso, em 18 tanques-rede $\left(1 \mathrm{~m}^{3}\right)$, com densidade de 25 peixes por tanque, em viveiro de terra de 0,1 ha. As seguintes variáveis foram avaliadas: peso, comprimento total, ganho de peso, biomassa, sobrevivência, rendimento de carcaça e filé, composição química dos filés, índices digestivo e hepatossomáticos, quociente intestinal. Não foram observadas diferenças no desempenho de crescimento. Peixes alimentados com dietas com casca de soja apresentaram maior quantidade de gordura e minerais nos filés. As fontes e níveis de fibra das dietas testadas não afetam o desempenho produtivo do jundiá.
\end{abstract}

Termos para indexação: Rhamdia quelen, carboidratos, fibra bruta, fibra em detergente neutro, tanques-rede.

\section{Rearing of jundiá fed with diets containing soy or cotton hull}

\begin{abstract}
The objective of this work was to evaluate the effect of crude fiber on growth, digestive parameters, and yield and carcass quality of jundiá (Rhamdia quelen) juveniles in net cages. The experimental design was completely randomized in a $2 \times 3$ factorial scheme (two sources - soy and cotton hull - and three levels of crude fiber: 4,7 and 10\%), with three replicates. Four hundred and fifty fishes $(129.11 \pm 7.8 \mathrm{~g})$ were randomly distributed in 18 net cages $\left(1 \mathrm{~m}^{3}\right)$, at densities of 25 fish per cage, in 0.1 ha earth fishpond. The following parameters were evaluated: weight, total length, weight gain, biomass, survival, carcass and fillet yield, chemical composition of fillet, digestive and hepato-somatic indexes, intestinal quotient. No difference was detected in the growth performance. Fish fed with soy hull diets presented more fat and mineral accumulation on fillets. The sources and fiber levels tested do not affect jundiá's performance.
\end{abstract}

Index terms: Rhamdia quelen, carbohydrates, crude fibre, neutral detergent fiber, net cages.

\section{Introdução}

O jundiá (Rhamdia quelen) é adaptado a diferentes ambientes e vem apresentando bons resultados em cultivo, principalmente em regiões mais frias. É um peixe de fácil reprodução, com boa resistência ao manejo e hábito alimentar onívoro, o que contribui para a aceitação de alimentos artificiais (Gomes et al., 2000; Barcellos et al., 2004; Carneiro \& Mikos, 2005). Além disso, apresenta excelente aceitação pelo mercado consumidor, tanto para pesca esportiva quanto para alimentação, com características propícias ao processamento (Carneiro, 2004).
A criação em tanques-rede viabiliza o cultivo dessa espécie em grandes reservatórios e locais de difícil implantação de sistemas convencionais. Esse sistema possui constante renovação de água e possibilita altas densidades de estocagem. Os peixes em tanques-rede são impossibilitados de procurar alimentos naturais que possam suprir alguma deficiência nutricional apresentada pela dieta fornecida, assim, todos os nutrientes exigidos pelos peixes devem estar contidos na ração oferecida (Carneiro, 2004).

Os ingredientes de origem vegetal vêm sendo utilizados como matéria-prima na formulação de rações para peixes, por terem composição química homogênea, 
facilidade de obtenção e preços mais baixos, quando comparados aos de origem animal. Porém a fração fibrosa existente nesses ingredientes pode intervir no aproveitamento do alimento pelos peixes, pois está diretamente ligada à digestão e absorção dos nutrientes (Hayashi et al., 2000; Lanna et al., 2004a). Para a tilápia (Oreochromis niloticus), níveis de fibra bruta entre 3,6 e $8,5 \%$ não interferem no desenvolvimento dos peixes (Anderson et al., 1984; Meurer et al., 2003; Lanna et al., 2004b). Outras espécies onívoras, como o pacu (Piaractus mesopotamicus) e a piracanjuba (Brycon orbignyanus), toleram níveis de até $9 \%$ de fibra bruta sem afetar o desempenho produtivo (Zanoni, 1996; Esquivel et al., 1999).

O objetivo deste trabalho foi avaliar o efeito da fibra bruta da dieta, no crescimento e nos parâmetros produtivos de juvenis de jundiá (Rhamdia quelen) criados em tanques-rede.

\section{Material e Métodos}

O experimento foi conduzido no Laboratório de Piscicultura da Universidade Federal de Santa Maria, de dezembro de 2005 a julho de 2006, com duração de 120 dias. Foram utilizados 18 tanques-rede de $1 \mathrm{~m}^{3}$ cada, dispostos em linha com $1 \mathrm{~m}$ de distância entre tanques, colocados em viveiro de terra com aproximadamente 0,1 ha e 1,7 m de profundidade, sem renovação de água mas com aeração mecânica (Aquapás). Cada tanquerede correspondia a uma unidade experimental. Foram utilizados 450 jundiás, todos de mesma desova, com peso médio inicial de $129,11 \pm 7,81 \mathrm{~g}$ e comprimento total inicial de $23,5 \pm 0,6 \mathrm{~cm}$. Foram distribuídos 25 peixes por unidade experimental, na densidade de $3,23 \mathrm{~kg} \mathrm{~m}^{-3}$. O período de adaptação aos tanques-rede e ao manejo diário, antes do início do experimento, foi de 21 dias.

Os tratamentos foram seis dietas isoprotéicas (32\%), com duas fontes (casca de soja e casca de algodão) e três níveis de fibra bruta $(4,7$ e 10\%) (Tabela 1). Foram utilizadas como fontes protéicas a farinha de carne e ossos e o farelo de soja (Lazzari et al., 2006).

A ração peletizada foi fornecida uma vez por dia, às $8 \mathrm{~h}$, na quantidade de $3 \%$ da biomassa por dia. A alimentação foi realizada com auxílio de comedouros localizados dentro de cada tanque-rede. A cada 20 dias,

Tabela 1. Composição das dietas experimentais (em percentagem) $)^{(1)}$.

\begin{tabular}{|c|c|c|c|c|c|c|}
\hline \multirow{2}{*}{ Ingrediente } & \multicolumn{3}{|c|}{ Casca de soja } & \multicolumn{3}{|c|}{ Casca de algodão } \\
\hline & $4 \%$ & $7 \%$ & $10 \%$ & $4 \%$ & $7 \%$ & $10 \%$ \\
\hline Farinha de carne e ossos & 30,00 & 30,00 & 30,00 & 30,00 & 30,00 & 30,00 \\
\hline Farelo de soja & 26,10 & 24,76 & 23,41 & 26,23 & 26,53 & 26,83 \\
\hline Farelo de arroz & 5,00 & 5,00 & 5,00 & 5,00 & 5,00 & 5,00 \\
\hline Milho triturado & 19,64 & 15,98 & 12,33 & 20,20 & 16,14 & 12,08 \\
\hline Óleo de soja & 3,00 & 3,00 & 3,00 & 3,00 & 3,00 & 3,00 \\
\hline Casca de soja & 4,74 & 12,74 & 20,74 & - & - & - \\
\hline Casca de algodão & - & - & - & 4,05 & 10,81 & 17,57 \\
\hline Material inerte (areia) & 6,00 & 3,00 & - & 6,00 & 3,00 & - \\
\hline Farinha de trigo & 2,00 & 2,00 & 2,00 & 2,00 & 2,00 & 2,00 \\
\hline Sal comum & 1,00 & 1,00 & 1,00 & 1,00 & 1,00 & 1,00 \\
\hline Fosfato bicálcico & 1,00 & 1,00 & 1,00 & 1,00 & 1,00 & 1,00 \\
\hline Vitaminas e minerais $^{(2)}$ & 1,50 & 1,50 & 1,50 & 1,50 & 1,50 & 1,50 \\
\hline Butil hidróxi tolueno (BHT) & 0,02 & 0,02 & 0,02 & 0,02 & 0,02 & 0,02 \\
\hline \multicolumn{7}{|l|}{ Composição centesimal ${ }^{(3)}$} \\
\hline Energia digestível $\left(\mathrm{kcal} \mathrm{kg}^{-1}\right)^{(4)}$ & $3.371,8$ & $3.383,8$ & $3.408,3$ & $3.304,1$ & $3.395,1$ & $3.417,9$ \\
\hline Umidade & 5,5 & 6,3 & 5,9 & 5,5 & 6,1 & 6,6 \\
\hline Proteína bruta & 33,6 & 33,9 & 34,4 & 33,0 & 33,9 & 34,6 \\
\hline Extrato etéreo & 10,0 & 9,8 & 9,6 & 9,3 & 9,9 & 9,6 \\
\hline Matéria mineral & 14,0 & 14,1 & 14,3 & 14,3 & 14,0 & 14,3 \\
\hline Extrativo não nitrogenado & 31,4 & 31,9 & 32,5 & 32,1 & 32 & 32,5 \\
\hline Fibra detergente neutro & 24,8 & 26,9 & 32,2 & 24,5 & 28,9 & 33,4 \\
\hline Fibra bruta & 5,0 & 7,3 & 9,2 & 5,3 & 7,2 & 9,0 \\
\hline
\end{tabular}

${ }^{(1)}$ Dietas ajustadas de acordo com Lazzari et al. (2006). ${ }^{(2)}$ Composição da mistura vitamínica e mineral (por kg de produto): ácido fólico, 400 mg; ácido nicotínico, $14.000 \mathrm{mg}$; ácido pantotênico, $8.000 \mathrm{mg}$; Co, $1.500 \mathrm{mg} ; \mathrm{Cu}, 15.000 \mathrm{mg}$; colina, $1.500 \mathrm{mg} ; \mathrm{Fe}, 50.000 \mathrm{mg} ; \mathrm{I}, 700 \mathrm{mg}$; Mn, $23.000 \mathrm{mg}$; Se, $250 \mathrm{mg}$; vit. A, 6.000.000 UI; vit. B1, $1.400 \mathrm{mg}$; vit. B2, $3.375 \mathrm{mg}$; vit. B6, $4.830 \mathrm{mg}$; vit. B12, 5.000 mg; vit. C, $25.000 \mathrm{mg}$; vit. D3, 530.000 UI; vit. E, $22.500 \mathrm{mg}$; vit. K3, $500 \mathrm{mg}$; Zn, $40.000 \mathrm{mg}$. ${ }^{(3)}$ Valores com base na matéria seca. ${ }^{(4)}$ Energia digestível $=[(\mathrm{PB} \times 5,65 \times 0,85)$ $+(\operatorname{EEx} 9,4 \times 0,9)+(\operatorname{ENN} \times 4,15 \times 0,7)] \times 10$. 
foi realizada a biometria e o ajuste da quantidade de ração oferecida. Semanalmente, foram monitorados os parâmetros físico-químicos da água do sistema de criação, como pH, alcalinidade, amônia total, nitrito e oxigênio, todos com kit colorimétrico (Alfakit), e a temperatura foi medida duas vezes por dia, com termômetro de bulbo de mercúrio.

$\mathrm{Na}$ avaliação dos parâmetros de carcaça, foram coletados seis peixes antes da biometria inicial. Aos 120 dias, foi coletado um peixe por unidade experimental (três por tratamento). Os peixes foram abatidos por punção na medula e, posteriormente, eviscerados e filetados. Com os filés, foi realizada a análise da composição centesimal. $O$ fígado e trato gastrintestinal foram pesados e medidos.

Os seguintes parâmetros foram avaliados: comprimento total (CT) - medida da porção anterior da cabeça até o final da nadadeira caudal, em $\mathrm{cm}$; peso (P) - obtido ao final do período, em g; ganho em peso (GP) diário, em g por dia; sobrevivência (S) percentagem de sobreviventes em relação ao número inicial de peixes, em cada tratamento; biomassa - obtida ao final do período, em g; rendimento de carcaça (RC) - diferença entre o peso inteiro e o peso eviscerado, com as brânquias, em percentagem (Melo et al., 2002); rendimento de filé (RF) (sem pele) - diferença entre o peso do filé e do peso vivo, em percentagem; índice hepatossomático (IHS) - obtido pelo quociente entre o peso do fígado e o peso do peixe inteiro; índice digestivosomático (IDS) - obtido pelo quociente entre o peso do trato digestório e o peso do peixe inteiro; quociente intestinal (QI) - obtido pelo comprimento do intestino dividido pelo comprimento do peixe.

Nos filés, a proteína bruta foi determinada pelo método de Kjeldahl com fator de conversão de 6,25. A matéria mineral e a umidade foram determinadas segundo Instituto Adolfo Lutz (1985). A fração lipídica foi determinada pelo método de Bligh \& Dyer (1959).

O delineamento experimental foi inteiramente casualizado, em arranjo fatorial (duas fontes de fibra bruta $\mathrm{x}$ três níveis de fibra bruta). Os dados foram submetidos ao teste de normalidade. Em seguida, foi realizada a análise de variância de duas vias, para avaliar o efeito das fontes de variação e sua interação. Os dados de níveis, quando significativos, foram submetidos à análise de regressão polinomial, e as médias das fontes foram comparadas pelo teste $\mathrm{t}$ de Student, a 5\% de probabilidade. Para a realização das análises estatísticas utilizou-se o SAS (SAS Institute, 1997).

\section{Resultados e Discussão}

Os parâmetros químicos de qualidade da água se mantiveram adequados para a espécie: $\mathrm{pH}, 7,62 \pm 0,2$; amônia total $\left(\mathrm{NH}_{3}+\mathrm{NH}_{4}\right), 0,26 \pm 0,5 \mathrm{ppm}$; nitrito $\left(\mathrm{NO}_{2}\right), 0,0 \mathrm{ppm}$; alcalinidade total $\left(\mathrm{CaCO}_{3}\right), 88,38 \pm 17,8 \mathrm{ppm}$; oxigênio dissolvido $\left.\left(\mathrm{O}_{2} \mathrm{D}\right), 6,31 \pm 0,8 \mathrm{ppm}\right)$. A temperatura foi o parâmetro de qualidade da água que mais variou durante o período experimental (Figura 1). A temperatura elevada da água $\left(34^{\circ} \mathrm{C}\right)$ do $20^{\circ}$ ao $60^{\circ}$ dia prejudicou efetivamente o ganho de peso dos animais, bem como o índice de sobrevivência. A partir do $60^{\circ}$ dia, a temperatura da água baixou à faixa tolerável, o que propiciou ganho de peso compensatório (Figura 2).

Um aumento na temperatura da água promove o crescimento, mas a partir de $31-32^{\circ} \mathrm{C}$ começam a ocorrer mortes dos alevinos (Baldisserotto \& Radünz Neto, 2004). Uma elevação na temperatura da água pode ser benéfica, mas se atingir valores acima da tolerada pela espécie, causa estresse e diminuição do consumo de alimento, o que afeta o crescimento e pode causar mortalidade. Não existem estudos que indicam a temperatura que promove o melhor rendimento do jundiá (Baldisserotto \& Radünz Neto, 2004).

Até o 60 으 dia de criação, não foi observado efeito $(p>0,05)$ das fontes e dos níveis de fibra estudados nas variáveis de crescimento: peso médio, comprimento total, biomassa, ganho de peso diário e sobrevivência. No entanto, pôde-se observar o efeito negativo de temperaturas elevadas no crescimento (Figura 2). Essas

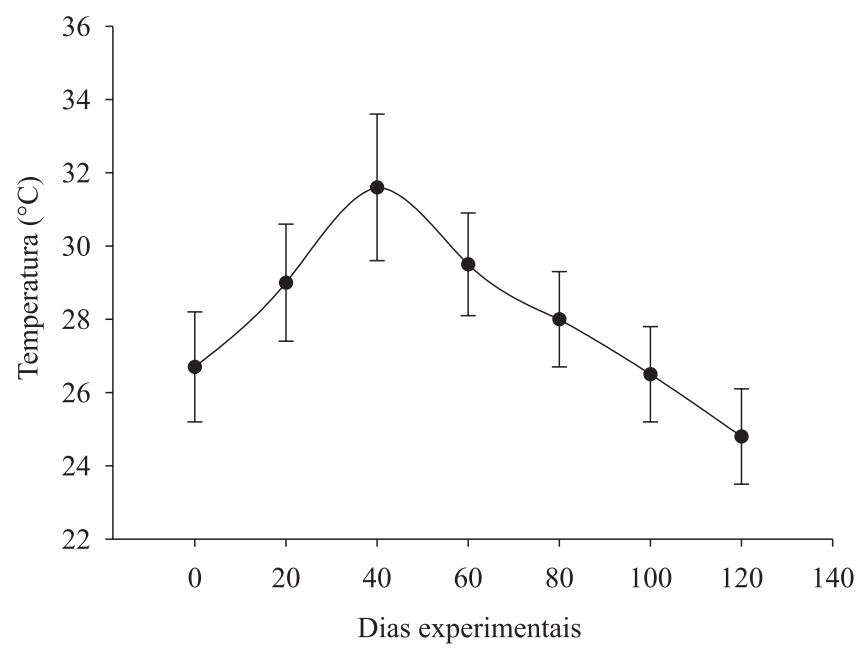

Figura 1. Temperatura da água, durante o experimento de criação de Rhamdia quelen em tanques-rede. 
variáveis não diferiram significativamente dos valores do início do experimento, o que torna preocupante o efeito do fator climático sobre esses resultados zootécnicos, do ponto de vista produtivo. Em temperaturas elevadas, o peixe encontra-se em condição de estresse. Nesse caso, o gasto energético para compensar esta situação é maior, e pode comprometer o crescimento.

Não foram observadas diferenças significativas, nas variáveis de crescimento $(\mathrm{p}>0,05)$ para os fatores níveis e fontes de fibra, aos 120 dias experimentais. Porém, foi possível observar a melhoria do desempenho entre os períodos de 0 a 60 dias e de 60 a 120 dias. O peso médio aumentou entre 50 e $60 \mathrm{~g}$. Em média, os peixes tiveram um ganho de $0,94 \mathrm{~g}$ por dia, semelhante ao valor obtido por Losekann (2006) - de 0,95 g por dia -, com 90 dias de criação da mesma espécie. A sobrevivência foi de $100 \%$ entre o 60 o e o 120 o dia.

A biomassa apresentou correlação positiva com os níveis de fibra bruta $(0,68)$ (Tabela 2); com isso, foi realizada a análise estatística com a sobrevivência como covariável, para excluir seu efeito sobre a biomassa. A partir do 60 - dia, o nível de $7 \%$ de fibra aumentou a biomassa $(\mathrm{p}<0,05)$. Não houve diferença para essa variável nas fontes de fibra estudadas.

Ao avaliar o crescimento de alevinos de tilápia (Oreochromis niloticus), Lanna et al. (2004b) não observaram redução no crescimento, com o nível de até 9\% de fibra bruta na dieta. Meurer et al. (2003) também

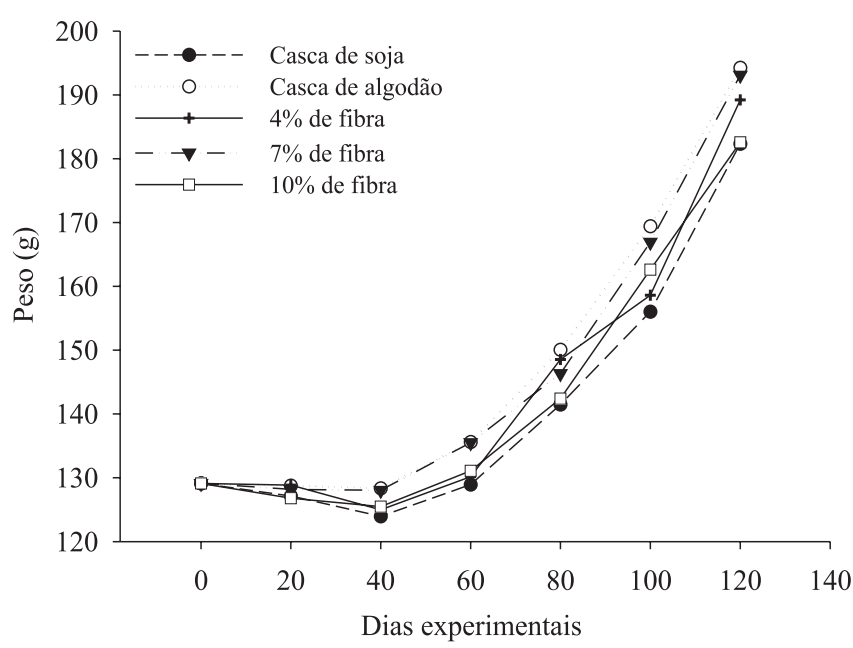

Figura 2. Crescimento de Rhamdia quelen no período experimental. observaram, para tilápia (Oreochromis niloticus), que níveis de até $8,5 \%$ de fibra bruta na alimentação não afetaram o desempenho.

Não houve diferença significativa entre os tratamentos quanto aos valores de rendimento de carcaça e de rendimento de filé (Tabela 3). Esses valores estão de acordo com os observados por Lazzari et al. (2006) e Losekann (2006), entre 80 e $87 \%$ de rendimento de carcaça e 30 e $32 \%$ de rendimento de filé de jundiá. $\mathrm{O}$ índice digestivo-somático e o quociente intestinal não apresentaram diferença significativa. Essas variáveis são indicativas de adaptação do trato gastrintestinal ao tipo de alimento ingerido. Conforme a composição dos ingredientes, o trato digestivo do peixe pode aumentar de tamanho e de peso (volume), na tentativa de aumentar a área de contato com o alimento e melhorar a

Tabela 2. Biomassa (g) e sobrevivência (\%) de juvenis de Rhamdia quelen, de 0 a 60 dias e de 60 a 120 dias, alimentados com duas fontes de fibra bruta, em três níveis ${ }^{(1)}$.

\begin{tabular}{|c|c|c|c|c|}
\hline \multirow[t]{2}{*}{ Tratamento } & \multicolumn{2}{|c|}{$0-60$ dias } & \multicolumn{2}{|c|}{$60-120$ dias } \\
\hline & Biomassa & Sobrevivência & Biomassa & Sobrevivência \\
\hline Casca de soja ${ }^{(2)}$ & $2.804,3$ & 94 & $3.783,1$ & 100 \\
\hline Casca de algodão ${ }^{(2)}$ & $2.948,2$ & 95 & $4.039,5$ & 100 \\
\hline $4 \%{ }^{(3)}$ & $2.855,8$ & 92 & $3.612,7$ & 100 \\
\hline $7 \%^{(3)}$ & $2.927,3$ & 97 & $4.255,3$ & 100 \\
\hline $10 \%^{(3)}$ & $2.845,8$ & 95 & $3.845,1$ & 100 \\
\hline $\mathrm{DPR}^{(4)}$ & 175,2 & 6,03 & 226,3 & - \\
\hline Nível (Ni) & ns & ns & $* *$ & - \\
\hline Fonte $(\mathrm{Ft})$ & ns & ns & ns & - \\
\hline $\mathrm{NixFt}$ & ns & ns & ns & - \\
\hline
\end{tabular}

${ }^{(1)}$ Biomassa inicial $=3.227,2$ g. ${ }^{(2)}$ Média dos níveis de fibra. ${ }^{(3)}$ Média das fontes de fibra. (4)Desvio-padrão residual. nsNão-significativo. $* *$ Significativo a $1 \%$ de probabilidade $\left(\mathrm{y}=363,2+1071,8 \mathrm{x}-72,2 \mathrm{x}^{2}\right.$; $\left.\mathrm{R}^{2}=68,14\right)$.

Tabela 3. Rendimento de carcaça (RC), rendimento de filé (RF), índice digestivo somático (IDS), índice hepatossomático (IHS), gordura visceral (GV) e quociente intestinal (QI) de juvenis de Rhamdia quelen alimentados com duas fontes de fibra bruta, em três níveis.

\begin{tabular}{|c|c|c|c|c|c|c|}
\hline \multirow[t]{2}{*}{ Tratamento } & $\mathrm{RC}$ & RF & IDS & IHS & $\mathrm{GV}$ & QI \\
\hline & \multicolumn{6}{|c|}{---------------------------(\%) } \\
\hline Casca de soja ${ }^{(1)}$ & 82,22 & 32,10 & 2,76 & 1,23 & 2,35 & 1,60 \\
\hline Casca de algodão ${ }^{(1)}$ & 80,67 & 31,35 & 3,21 & 1,32 & 2,91 & 1,51 \\
\hline $4 \%(2)$ & 81,07 & 32,02 & 3,28 & 1,35 & 2,88 & 1,68 \\
\hline $7 \%{ }^{(2)}$ & 81,30 & 31,60 & 2,85 & 1,28 & 1,75 & 1,39 \\
\hline $10 \%^{(2)}$ & 81,97 & 31,56 & 2,82 & 1,19 & 3,26 & 1,59 \\
\hline $\mathrm{DPR}^{(3)}$ & 4,55 & 2,67 & 0,45 & 0,21 & 1,59 & 0,27 \\
\hline Nível (Ni) & $\mathrm{ns}$ & $\mathrm{ns}$ & $\mathrm{ns}$ & $\mathrm{ns}$ & ns & ns \\
\hline Fonte $(\mathrm{Ft})$ & ns & $\mathrm{ns}$ & $\mathrm{ns}$ & ns & ns & ns \\
\hline $\mathrm{NixFt}$ & $\mathrm{ns}$ & $\mathrm{ns}$ & $\mathrm{ns}$ & $\mathrm{ns}$ & ns & ns \\
\hline
\end{tabular}

(1)Média dos níveis de fibra. ${ }^{(2)}$ Média das fontes de fibra. ${ }^{(3)}$ Desvio-padrão

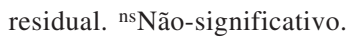


digestibilidade (Leenhouwers et al., 2006). Porém, não foi observado efeito do aumento do nível de fibra e nem das fontes estudadas nessas variáveis (Tabela 3). Não foi observada, também, diferença para o índice hepatossomático. Possivelmente, o período de 120 dias, deste trabalho, foi insuficiente para que esses índices mostrassem resposta ao efeito dos níveis de fibra. A gordura visceral é um fator que afeta diretamente o rendimento de carcaça e aumenta o volume das vísceras; essa variável não diferiu entre fontes e níveis estudados, e os valores foram semelhantes aos encontrados por Webster et al. (1995), em cultivo de "blue catfish" (Ictalurus furcatus) em tanque-rede.

Não houve diferença significativa quanto à umidade e à quantidade de proteína bruta nos filés, aos 120 dias de criação (Tabela 4). Esse resultado está de acordo com Lanna et al. (2004b), que também não observaram o efeito de níveis crescentes de fibra bruta na dieta de tilápias na composição química da carcaça. Não foi observado efeito do nível de fibra na quantidade de matéria mineral e de lipídios, entretanto, houve influência das fontes de fibra testadas. Os filés dos peixes alimentados com rações com casca de soja tiveram maior quantidade de matéria mineral no filé. Isso pode ser explicado pela diferença na composição da fração fibrosa dos diferentes ingredientes. A casca de algodão possui mais lignina que a casca de soja, e a lignina tem forte capacidade de se complexar com íons metálicos, o que impossibilita a absorção desses pelo epitélio (Hayashi et al., 2000). Esse fato pode ter causado menor acúmulo de minerais no filé dos peixes alimentados com esse ingrediente.

Tabela 4. Composição centesimal (\%) dos filés de juvenis de Rhamdia quelen, alimentados com duas fontes de fibra bruta, em três níveis ${ }^{(1)}$.

\begin{tabular}{lcccc}
\hline Tratamento & Umidade & Proteína & Cinzas & Lipídios \\
\hline Casca de soja $^{(2)}$ & 75,19 & 17,61 & $1,74 \mathrm{a}$ & $7,22 \mathrm{a}$ \\
Casca de algodão $^{(2)}$ & 74,69 & 17,20 & $1,51 \mathrm{~b}$ & $5,38 \mathrm{~b}$ \\
$4 \%{ }^{(3)}$ & 74,24 & 17,55 & 1,63 & 6,28 \\
$7 \%^{(3)}$ & 75,57 & 17,66 & 1,53 & 6,42 \\
$10 \%{ }^{(3)}$ & 75,00 & 17,02 & 1,70 & 6,22 \\
\hline DPR $^{(4)}$ & 1,65 & 1,80 & 0,20 & 1,45 \\
\hline Nível $(\mathrm{Ni})$ & $\mathrm{ns}$ & $\mathrm{ns}$ & $\mathrm{ns}$ & $\mathrm{ns}$ \\
Fonte $(\mathrm{Ft})$ & $\mathrm{ns}$ & $\mathrm{ns}$ & $*$ & $*$ \\
NixFt & $\mathrm{ns}$ & $\mathrm{ns}$ & $\mathrm{ns}$ & $\mathrm{ns}$ \\
\hline
\end{tabular}

${ }^{(1)}$ Expresso na matéria natural. ${ }^{(2)}$ Média dos níveis de fibra; nos casos em que as diferenças foram significativas, as médias foram comparadas pelo teste t de Student, a 5\% de probabilidade. ${ }^{(3)}$ Média das fontes de fibra. ${ }^{(4)}$ Desvio-padrão residual. ns Não-significativo. ${ }^{*}$ Significativo a $5 \%$ de probabilidade.
Os peixes alimentados com a casca de soja também apresentaram maior quantidade de lípidios no filé $(\mathrm{p}<0,05)$. A casca de soja, em comparação com a casca de algodão, possui grande quantidade de substâncias pécticas na sua fração fibrosa, que tem elevado grau de fermentação por microrganismos do trato gastrintestinal (Van Soest et al., 1991; Zambom et al., 2001; Silva et al., 2004), e isto propicia maior produção de ácidos graxos voláteis (AGV) no intestino. Esses são utilizados como fonte de energia pelo epitélio, o que influencia a absorção e a deposição de gordura no organismo do animal (Bach Knudsen, 2001).

\section{Conclusões}

1. As fontes e os níveis de fibra bruta, testados nas dietas, não afetam o desempenho produtivo, os índices digestivos e o rendimento de carcaça e de filé de jundiás.

2. Os peixes alimentados com casca de soja, como fonte fibrosa, apresentam maior quantidade de lipídios no filé.

\section{Agradecimentos}

À Coordenação de Aperfeiçoamento de Pessoal de Ensino Superior, por concessão de bolsa; à Seap/MCT/ Fundo setorial Agronegócio/Fundo CT Hidro/Finep, pelo apoio financeiro; às empresas Giovelli \& Com. Ltda. e Vitagri Nutrição Animal, pelo fornecimento de ingredientes.

\section{Referências}

ANDERSON, J.; JACKSON, A.J.; MATTY, A.J.; CAPPER, B.S. Effects of dietary carbohydrate and fibre on the tilapia (Oreochromis niloticus). Aquaculture, v.37, p.303-314, 1984.

BACH KNUDSEN, K.E. The nutritional significance of "dietary fibre" analysis. Animal Feed Science and Technology, v.90, p.3-20, 2001.

BALDISSEROTTO, B.; RADÜNZ NETO, J. Criação de Jundiá. Santa Maria: Ed. UFSM, 2004. 232p.

BARCELLOS, L.J.G.; KREUTZ, C.; QUEVEDO, R.M.; FIOREZE, I.; CERICATO, L.; SOSO, A.B.; FAGUNDES, M.; CONRAD, J.; BALDISSERA, R.K.; BRUSCHI, A.; RITTER, F. Nursery rearing of jundiá, Rhamdia quelen (Quoy \& Gaimard) in cages: cage type, stocking density and stress response to confinement. Aquaculture, v.232, p.383-394, 2004.

BLIGH, E.G.; DYER, W.J. A rapid method of total lipid extraction and purification. Canadian Journal of Biochemistry and Physiology, v.37, p.911-917, 1959.

CARNEIRO, P.C.F. A produção do jundiá em cativeiro. In: BALDISSEROTTO, B.; RADÜNZ NETO, J. Criação de jundiá. Santa Maria: UFSM, 2004. p.117-141. 
CARNEIRO, P.C.F.; MIKOS, J.D. Freqüência alimentar e crescimento de alevinos de jundiá, Rhamdia quelen. Ciência Rural, v.35, p.187-191, 2005.

ESQUIVEL, J.R.; PEZZATO, L.E.; ZANIBONI FILHO, E.; VICENTINI, C.A. Utilização da fibra bruta na nutrição do piracanjuba (Brycon orbignyanus). Acta Scientiarum, v.21, p.725-731, 1999.

GOMES, L.C.; GOLOMBIESKI, J.I.; CHIPPARI-GOMES, A.R.; BALDISSEROTTO, B. Biologia do jundiá Rhamdia quelen (Teleostei, Pimelodidae). Ciência Rural, v.30, p.179-185, 2000.

HAYASHI, C.; MEURER, F.; BOSCOLO, W.R.; SOARES, C.M. Fontes de fibra bruta em dietas de alevinos de tilápia-do-nilo (Oreochromis niloticus). Acta Scientiarum, v.22, p.689-694, 2000.

INSTITUTO ADOLFO LUTZ. Normas analíticas do Instituto Adolfo Lutz: métodos químicos e físicos para análise de alimentos. 3.ed. São Paulo: IAL, 1985. v.1. 368p.

LANNA, E.A.T.; PEZZATO, L.E.; CECON, P.R.; FURUYA, W.M.; BOMFIM, M.A.D. Digestibilidade aparente e trânsito gastrintestinal em tilápia-do-nilo (Oreochromis niloticus), em função da fibra bruta da dieta. Revista Brasileira de Zootecnia, v.33, p.2186-2192, 2004a.

LANNA, E.A.T.; PEZZATO, L.E.; FURUYA, W.M.; VICENTINI, C.A.; CECON, P.R.; BARROS, M.M. Fibra bruta e óleo em dietas práticas para alevinos de tilápia-do-nilo (Oreochromis niloticus). Revista Brasileira de Zootecnia, v.33, p.2177-2185, 2004b.

LAZZARI, R.; RADÜNZ NETO, J.; EMANUELLI, T.; PEDRON, F.A.; COSTA, M.L.; LOSEKANN, M.E.; CORREIA, V.; BOCHI, V.C. Diferentes fontes protéicas para a alimentação do jundiá (Rhamdia quelen). Ciência Rural, v.36, p.240-246, 2006.

LEENHOUWERS, J.I.; ADJEI-BOATENG, D.; VERRETH, J.A.J.; SCHRAMA, J.W. Digesta viscosity, nutrient digestibility and organ weights in African catfish (Clarias gariepinus) fed diets supplemented with different levels of a soluble non-starch polysaccharide. Aquaculture Nutrition, v.12, p.111-116, 2006.
LOSEKANN, M.E. Produção de jundiá (Rhamdia quelen) alimentado com dietas contendo óleos de arroz, canola ou soja. 2006. 44p. Dissertação (Mestrado) - Universidade Federal de Santa Maria, Santa Maria.

MELO, J.F.B.; RADÜNZ NETO, J.; SILVA, J.H.S.; TROMBETTA, C.G. Desenvolvimento e composição corporal de alevinos de jundiá (Rhamdia quelen) alimentados com dietas contendo diferentes fontes de lipídios. Ciência Rural, v.32, p.323-327, 2002.

MEURER, F.; HAYASHI, C.; BOSCOLO, W.R. Fibra bruta para alevinos de tilápia-do-nilo (Oreochromis niloticus). Revista Brasileira de Zootecnia, v.32, p.256-261, 2003.

SAS INSTITUTE. Statistical Analysis System: user's guide: version 6.08. 4.ed. Cary: SAS, 1997. 846p.

SILVA, D.C.; KAZAMA, R.; FAUSTINO, J.O.; ZAMBOM, M.A.; SANTOS, G.T.; BRANCO, A.F. Digestibilidade in vitro e degradabilidade in situ da casca do grão de soja, resíduo de soja e casca de algodão. Acta Scientiarum, v.26, p.501-506, 2004.

VAN SOEST, P.J.; ROBERTSON, J.B.; LEWIS, B.A. Methods for dietary fiber, neutral detergent fiber, and nonstarch polysaccharides in relation to animal nutrition. Journal of Dairy Science, v.74, p.3583-3597, 1991.

WEBSTER, C.D.; TIU, L.G.; TIDWELL, J.H.; REED, E.B. Effects of feeding the repartitioning agent L644,969 on growth and body composition of blue catfish, Ictalurus furcatus, fed diets containing two protein levels reared in cages. Aquaculture, v.134, p.247-256, 1995.

ZAMBOM, M.A.; SANTOS, G.T.; MODESTO, E.C.; ALCADE, C.R.; GONÇALVES, G.D.; SILVA, D.C.; SILVA, K.T.; FAUSTINO, J.O. Valor nutricional da casca de soja, farelo de soja, milho moído e farelo de trigo para bovinos. Acta Scientiarum, v.23, p.937-943, 2001.

ZANONI, M.A. Níveis de fibra bruta em dietas de crescimento do pacu (Piaractus mesopotamicus) Holmberg, 1887. 1996. 66p. Dissertação (Mestrado) - Universidade Federal de Santa Catarina, Florianópolis.

Recebido em 8 de maio de 2007 e aprovado em 4 de dezembro de 2007 